\title{
Direct molecular evidence for both multicentric and monoclonal carcinogenesis followed by transdifferentiation from hepatocellular carcinoma to cholangiocarcinoma in a case of metachronous liver cancer
}

\author{
SUMIE OHNI $^{1}$, HIROMI YAMAGUCHI ${ }^{2}$, YUKARI HIROTANI ${ }^{1}$, YOKO NAKANISHI ${ }^{1}$, \\ YUTAKA MIDORIKAWA ${ }^{3}$, MASAHIKO SUGITANI ${ }^{1}$, HIROMU NARUSE ${ }^{1}$, \\ TOMOHIRO NAKAYAMA ${ }^{1}$, MAKOTO MAKISHIMA ${ }^{2}$ and MARIKO ESUMI ${ }^{2}$ \\ ${ }^{1}$ Department of Pathology and Microbiology; ${ }^{2}$ Division of Biochemistry, Department of Biomedical Sciences; \\ ${ }^{3}$ Department of Surgery, Nihon University School of Medicine, Tokyo 173-8610, Japan
}

Received April 23, 2021; Accepted October 18, 2021

DOI: $10.3892 / \mathrm{ol} .2021 .13140$

\begin{abstract}
Frequent recurrence is a major issue in liver cancer and histological heterogeneity frequently occurs in this cancer type. However, it has remained elusive whether such cancers are multicentric or monoclonal. To elucidate the clonal evolution of hepatocellular carcinoma (HCC) recurrence and combined hepatocellular-cholangiocarcinoma (cHCC-CCA) development, the somatic mutation frequency and signatures in a patient with triple occurrence of liver cancer every three years were examined, with samples designated as \#1HCC, \#2HCC and \#3cHCC-CCA, respectively. A total of four tumor regions, including $\mathrm{HCC}(\# 3 \mathrm{HCC})$ and intrahepatic $\mathrm{CCA}$
\end{abstract}

Correspondence to: Professor Mariko Esumi or Professor Makoto Makishima, Division of Biochemistry, Department of Biomedical Sciences, Nihon University School of Medicine, 30-1 Ohyaguchikami-cho, Itabashi-ku, Tokyo 173-8610, Japan

E-mail: esumi.mariko@nihon-u.ac.jp

E-mail: makishima.makoto@nihon-u.ac.jp

Abbreviations: $\mathrm{HCC}$, hepatocellular carcinoma; HBV, hepatitis B virus; $\mathrm{HCV}$, hepatitis $\mathrm{C}$ virus; $\mathrm{IM}$, intrahepatic metastasis; $\mathrm{MC}$, multicentric carcinogenesis; cHCC-CCA, combined hepatocellularcholangiocarcinoma; iCCA, intrahepatic cholangiocarcinoma; HPCs, hepatic stem or progenitor cells; LCM, laser capture microdissection; qPCR, quantitative PCR; dPCR, digital PCR; FFPE, formalin-fixed, paraffin-embedded; SNV, single-nucleotide variant; GAPDH, glyceraldehyde-3-phosphate dehydrogenase; KMT2D, histone-lysine N-methyltransferase 2D; TP53, tumor

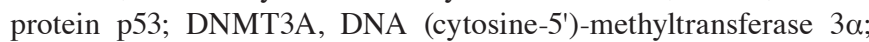
PKHD1, polycystic kidney and hepatic disease 1; TLR4, toll like receptor 4

Key words: metachronous cancer, cancer evolution, intrahepatic metastasis, multicentric carcinogenesis, hepatocellular carcinoma, combined hepatocellular-cholangiocarcinoma, transdifferentiation, laser capture microdissection, next-generation sequencing
(\#3iCCA) components of \#3cHCC-CCA, and three nontumor regions (\#1N, \#2N and \#3N) were precisely dissected from formalin-fixed paraffin-embedded tissues of each surgical specimen. DNA was extracted and subjected to tumor-specific somatic mutation determination. Of note, five nonsynonymous single-nucleotide variants (SNVs), namely those of KMT2D, TP53, DNMT3A, PKHD1 and TLR4, were identified in \#3cHCC-CCA. All five SNVs were detected in both \#3HCC and \#3iCCA and \#2HCC but not in \#1HCC. The telomerase reverse transcriptase (TERT) promoter mutation C228T, but not C250T, was observed in all tumors. Digital PCR of C228T also indicated the presence of the TERT promoter mutation C228T in nontumorous liver tissues (\#1N, \#2N and \#3N) at a frequency of $0.11-0.83 \%$ compared with normal liver and blood samples. These results suggest the following phylogenetic evolution of three metachronous liver cancers: \#1HCC was not related to \#2HCC, \#3HCC and \#3iCCA; both \#3HCC and \#3iCCA arose from \#2HCC. From the above, three novel findings were deduced: i) Both multicentric occurrence and intrahepatic metastasis may be involved in liver cancer in a three-year interval; ii) transdifferentiation from HCC to iCCA is a possible pathogenic mechanism of cHCC-CCA; and iii) a nontumorous, noncirrhotic liver may contain a preneoplastic region with a cancer driver mutation in the TERT promoter.

\section{Introduction}

Hepatocellular carcinoma (HCC) is one of the most common types of primary malignant tumor. HCC usually develops in the background of chronic liver diseases such as chronic hepatitis or liver cirrhosis caused by hepatitis B virus (HBV) or hepatitis $\mathrm{C}$ virus (HCV) infection, alcohol intake or metabolic syndrome (1). The challenging problem of HCC is its easy recurrence after curative resection; indeed, the recurrence rate within 5 years is $70 \%$ (2). There are two different mechanisms of HCC recurrence: Intrahepatic metastasis (IM) of primary $\mathrm{HCC}$ and multicentric carcinogenesis (MC) of HCC independent of primary HCC. Several discriminating 
factors of IM and MC have been identified and reviewed $(3,4)$. IM is characterized by early recurrence of HCC (e.g., within 2 years), pathological similarity and a more advanced grade of HCC. MC is characterized by late recurring HCC, different pathological features and relatively early-stage HCC. However, despite the clinical importance of discrimination in treatment and prognosis, it is difficult to precisely discriminate these two types of recurrence. Recently, molecular omics approaches, such as genomics, transcriptomics, proteomics and metabolomics, have been used to discriminate between MC and IM $(3,5)$.

A similar challenging issue regarding carcinogenesis exists in combined hepatocellular-cholangiocarcinoma (cHCC-CCA). cHCC-CCA is a primary liver carcinoma with unequivocal features of both hepatic and cholangiocytic differentiation within the same tumor based on morphology revealed by hematoxylin and eosin staining (6). There are three possible models for the origin and evolution of HCC and intrahepatic CCA (iCCA) components in cHCC-CCA: i) Hepatocytes and cholangiocytes synchronously transform to $\mathrm{HCC}$ and iCCA, respectively, even in the same nodule; ii) HCC develops first and then partial HCC transdifferentiates into iCCA in the same nodule (6-8); iii) hepatic stem or progenitor cells (HPCs) expand to progenitor-like tumors and then synchronously differentiate into HCC and iCCA in the same nodule. HPC directly develops into cHCC-CCA via a single-cell origin $(6,8,9)$. The first model involves a different origin of carcinoma, whereas the second and third models involve the same origin. Molecular analysis, such as genomics and transcriptomics, is useful for clarifying the evolution of cHCC-CCA $(10,11)$.

To clarify the cancer origin and evolution of the two issues described above (i.e., the mechanism of HCC recurrence and the origin of cHCC-CCA), it is necessary to precisely separate cancer samples to be used for mutational profiling, including mutation frequency determination. In the present study, a case of triple occurrence of liver cancer with a three-year interval was examined; the first and second liver cancers were diagnosed as HCC; the third was diagnosed as cHCC-CCA. To precisely separate cancer samples, laser capture microdissection (LCM) of sample tissues was performed and they were histopathologically diagnosed. To obtain precise mutational profiles, next-generation sequencing (NGS) of 409 cancer-associated genes was performed and the mutation frequency was then quantified by allele-specific quantitative PCR (qPCR) and digital PCR (dPCR). The two aspects of the phylogenetic tree of liver cancer components were analyzed as follows: i) Whether the three metachronous liver cancers were of the same origin; and ii) whether the two synchronous components of cHCC-CCA, i.e., HCC and iCCA, were of the same origin.

\section{Materials and methods}

Liver tissue samples and DNA extraction. The patient was a 71-year-old male with a primary HCC (\#1HCC) in 2007 who subsequently developed two recurrent liver cancers every three years; the second was HCC (\#2HCC) and the third was cHCC-CCA (\#3cHCC-CCA). Noncancerous regions of \#1N ( $2 \mathrm{~cm}$ away from the tumor), \#2N (1 cm from the tumor) and
\#3N (2 cm from the tumor) exhibited chronic hepatitis with a fibrosis score of F2. The patient was consistently positive for anti-HCV and hepatitis B surface (HBs) antibodies and negative for serum HBV DNA (Table SI) and received curative resection as a standard clinical treatment after each diagnosis. The patient had no history of alcohol abuse and did not receive any antiviral therapy for $\mathrm{HCV}$ infection. Formalin-fixed, paraffin-embedded (FFPE) liver tissues collected during surgery for pathological diagnosis were sliced into thin sections of $10 \mu \mathrm{m}$ in thickness. LCM was performed with a PALM-MBIII-N (Carl Zeiss AG) to obtain \#3HCC and \#3iCCA lesions (Fig. 1). Macrodissection was performed to obtain \#1HCC, \#2HCC and \#3cHCC-CCA lesions (Fig. 1). Nontumorous tissue sections (\#1N, \#2N and \#3N) were also obtained from the terminal FFPE tissues of resected specimens. DNA was extracted from the FFPE samples using the RecoverAll Total Nucleic Acid Isolation Kit for FFPE (Thermo Fisher Scientific, Inc.) with certain modifications as described previously $(12,13)$. DNA was also extracted from fresh-frozen liver specimens, nontumorous liver of a patient with liver metastasis of colon cancer, to use as controls for TaqMan quantitative PCR (qPCR) $(12,13)$. The present study was approved by the Ethics Committee of Nihon University School of Medicine (approval no. 237-1). Informed consent was obtained from the patients prior to the start of the study.

TaqMan $q P C R$. Quality assessment of FFPE DNA was performed by TaqMan qPCR of glyceraldehyde-3-phosphate dehydrogenase (GAPDH) and the ratio of FFPE DNA quality to frozen tissue DNA was calculated by the $\Delta$ quantification cycle $(\Delta \mathrm{Cq})$ method, as described previously $(12,13)$; PCR was performed in a $10-\mu 1$ reaction mixture containing Premix Ex Taq (Probe qPCR; Takara Bio, Inc.) with an initial denaturation step at $95^{\circ} \mathrm{C}$ for $20 \mathrm{sec}$, followed by 45 cycles at $95^{\circ} \mathrm{C}$ for $1 \mathrm{sec}$ and $60^{\circ} \mathrm{C}$ for $20 \mathrm{sec}$ using StepOnePlus (Thermo Fisher Scientific, Inc.). HBV-DNA was also quantified using a TaqMan Gene Expression Assay (Thermo Fisher Scientific, Inc.) and QuantStudio 3 System (Thermo Fisher Scientific, Inc.). Assay IDs for GAPDH and HBV DNA are presented in Table SII. All assays were performed in duplicate.

Comprehensive Cancer Panel (CCP) amplicon sequencing. DNA samples from \#3cHCC-CCA and \#3N were subjected to amplicon sequencing using Ion AmpliSeq Comprehensive Cancer Panel (Thermo Fisher Scientific, Inc.), as described previously (13). Candidates for cancer (\#3cHCC-CCA)-specific single-nucleotide variants (SNVs) were identified by Tumor-Normal Pair Analysis version 5.2 of Ion Reporter (https://ionreporter.thermofisher.com/ir/).

SYBR green allele-specific $q P C R$. Mutation frequency was determined by allele-specific qPCR, as described previously (13); allele-specific qPCR was quantitatively performed using THUNDERBIRD SYBR qPCR Mix (Toyobo Life Science) with the QuantStudio 3 System (Thermo Fisher Scientific, Inc.). PCR was performed in a $10-\mu 1$ reaction in duplicate by preheating at $95^{\circ} \mathrm{C}$ for $10 \mathrm{~min}$, followed by 45 cycles of $95^{\circ} \mathrm{C}$ for $15 \mathrm{sec}$ and 60 to $64^{\circ} \mathrm{C}$ for $60 \mathrm{sec}$. Allele-specific primers for wild-type $(\mathrm{Wt})$ and mutant $(\mathrm{Mu})$ sequences and a single opposite-directed primer were designed 


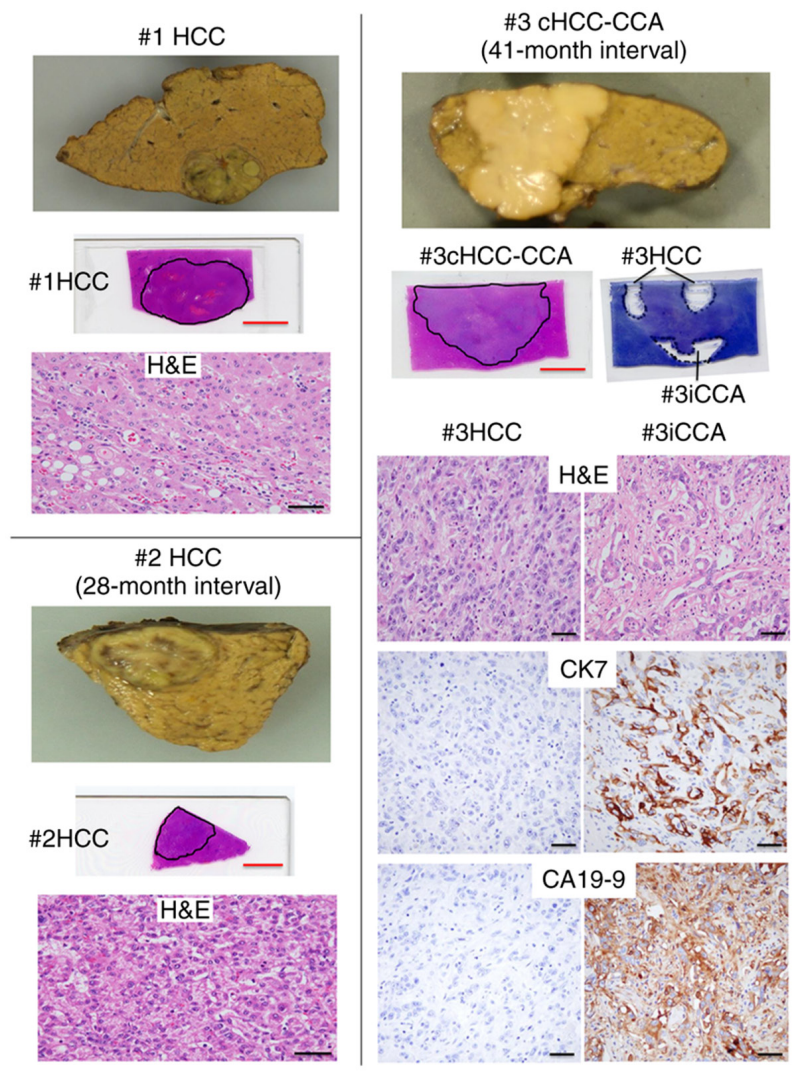

Figure 1. Gross and histological features of three metachronous liver cancers curatively resected every three years (\#1 to \#3, respectively). \#1, well-differentiated HCC, $2.6 \times 1.7 \mathrm{~cm}$ in diameter; \#2, moderately differentiated $\mathrm{HCC}, 1.8 \times 1.4 \mathrm{~cm}$ in diameter; \#3, cHCC-CCA, $4.5 \times 4.5 \mathrm{~cm}$ in diameter The outlined areas were macroscopically dissected for \#1HCC, \#2HCC and \#3cHCC-CCA and laser capture microdissected for \#3HCC and \#3CCA after staining with toluidine blue (red scale bars, $1 \mathrm{~cm}$ ). DNA was then extracted. Histological features of all cancerous regions are presented by $\mathrm{H} \& \mathrm{E}$ staining (scale bars, $50 \mu \mathrm{m}$ ). \#3cHCC-CCA was subjected to immunohistochemica staining for CK7 and CA19-9 and each result for the \#3HCC and \#3iCCA regions is presented (scale bars, $50 \mu \mathrm{m}$ ). HCC, hepatocellular carcinoma; cHCC-CCA, combined hepatocellular-cholangiocarcinoma; iCCA, intrahepatic cholangiocarcinoma; CK7, cytokeratin 7; CA19-9, carbohydrate/cancer antigen 19-9; H\&E, hematoxylin and eosin.

as previously described (13) and as presented in Table SII, together with each annealing temperature. The ratio of the $\mathrm{Mu}$ allele to the $\mathrm{Wt}$ allele was calculated as $2^{-\Delta \mathrm{Cq}}$, where $\Delta \mathrm{Cq}$ is the result of subtracting the $\mathrm{Cq}$ value of the PCR for the $\mathrm{Wt}$ from that of the $\mathrm{Mu}$. The $\mathrm{Mu}$ allele frequency was calculated as $2^{-\Delta \mathrm{Cq}} /\left(1+2^{-\Delta \mathrm{Cq}}\right)$, as described previously (13). The Mu allele frequency was used for cluster analysis of cancers with the agglomerative clustering method and the phylogenetic tree was constructed using the Ward method (14). To determine the Mu cell population, the two-hit theory of the mutation was hypothesized, whereby all Mu cells are heterozygous with the first-hit SNV when the Mu allele frequency is $<0.5$ and all cells consist of $\mathrm{Mu}$ heterozygotes and hemizygotes, which undergo Wt allele loss as the second hit when the Mu allele frequency is $>0.5$. In the former case, the Mu heterozygote population was calculated by the equation of heterozygote population $=2 \mathrm{x}$ allele frequency. In the latter case, the populations of Mu heterozygotes and hemizygotes were calculated by the equations of $\mathrm{Mu}$ heterozygotes population=1/allele frequency- 1 and hemizygote population=2-1/allele frequency, respectively.
TERT promoter mutation. TERT promoter mutations (C228T, C225T) were analyzed by Sanger sequencing of nested PCR products. Nested PCR amplification of the TERT promoter was performed using PrimeSTAR GXL DNA polymerase (Takara Bio, Inc.) and the first primer pair F1-R1 followed by the second primer pair F2-R2 (Table SII). The reaction conditions were as follows: $94^{\circ} \mathrm{C}$ for $1 \mathrm{~min}$ and 40 cycles of $98^{\circ} \mathrm{C}$ for $10 \mathrm{sec}$ and $68^{\circ} \mathrm{C}$ for $1 \mathrm{~min}$. Of the first PCR mix (20 $\mu \mathrm{l}), 1 \mu \mathrm{l}$ was used in the $20-\mu 1$ reaction for the second PCR. Sanger sequencing of the products from the second PCR was performed as previously described (13).

dPCR of the TERT promoter mutation C228T was performed using the TaqMan dPCR Liquid Biopsy Assay TERT_C228T (Hs000000092_rm; Thermo Fisher Scientific, Inc.) with the QuantStudio 3D Digital PCR System (Thermo Fisher Scientific, Inc.). PCR was performed using 60 to $1,000 \mathrm{ng}$ of sample DNA, with the following reaction conditions: $96^{\circ} \mathrm{C}$ for $10 \mathrm{~min}, 54$ cycles of $55^{\circ} \mathrm{C}$ for $2 \mathrm{~min}$ and $98^{\circ} \mathrm{C}$ for $30 \mathrm{sec}$, and two holds of $55^{\circ} \mathrm{C}$ for $2 \mathrm{~min}$, followed by $10^{\circ} \mathrm{C}$ indefinitely. The ramp rate of each temperature change was $1.6^{\circ} \mathrm{C} / \mathrm{sec}$, according to the manufacturer's instruction. The dPCR data were analyzed using QuantStudio 3D AnalysisSuite version 3.1 (Thermo Fisher Scientific, Inc.). Reproducibility was confirmed using two different concentrations of DNA from three representative samples. The negative controls consisted of two DNA samples, one from nontumorous liver tissue (normal liver) of a patient with liver metastasis of gallbladder cancer and another from the blood of a healthy subject using $60 \mathrm{ng}$ DNA/reaction. The mutation frequency was used for the cluster analysis of cancers as mentioned above, together with the mutant allele frequency of other genes. Informed consent was obtained from these subjects.

Immunohistochemistry. Sections of FFPE tissues $(4 \mu \mathrm{m})$ were treated with $10 \mathrm{mM}$ citrate buffer $\left(\mathrm{pH} \mathrm{6.0)}\right.$ ) at $120^{\circ} \mathrm{C}$ for $15 \mathrm{~min}$ for antigen retrieval. After quenching of endogenous peroxidase with $3 \%$ hydrogen peroxide for $5 \mathrm{~min}$ at room temperature. The sections were incubated with a 100 -fold diluted mouse monoclonal anti-cytokeratin (CK)7 antibody (clone OV-TL 12/30; catalog no. M7018; Agilent Technologies, Inc.) or 50-fold diluted mouse monoclonal anti-carbohydrate/cancer antigen (CA)19-9 antibody (clone 1116-NS-19-9; catalog no. M3517; Agilent Technologies, Inc.) for $1 \mathrm{~h}$ at room temperature. The sections were then incubated with Histofine Simple Stain MAX-PO (MULTI; Nichirei Biosciences) as secondary antibody for $30 \mathrm{~min}$ at room temperature, visualized with 3,3'-diaminobenzidine chromogen (Wako Pure Chemical Industries) and counterstained with hematoxylin. The proportion of positive cells in the HCC and iCCA area was determined by measurement of the positive area in five fields using cellSens Dimension 1.16 (Olympus Corporation).

\section{Results}

Histological findings for three metachronous liver cancers. In the present case, three metachronous liver cancers developed with intervals of 28 and 41 months. The first and second liver cancers were diagnosed as HCC (\#1HCC, \#2HCC) and the third as cHCC-CCA (\#3cHCC-CCA) (Fig. 1). All of the metachronous liver cancers were of the nodular type (Fig. 1). The 
Table I. Allele frequency of five SNVs in five samples from three metachronous liver cancers.

\begin{tabular}{|c|c|c|c|c|}
\hline \multirow[b]{2}{*}{$\mathrm{SNV}^{\mathrm{a}} / \mathrm{sample}^{\mathrm{b}}$} & \multicolumn{2}{|c|}{ Allele frequency } & \multicolumn{2}{|c|}{ Mutant cell population } \\
\hline & $\mathrm{CCP}^{\mathrm{c}}$ & $\mathrm{qPCR}^{\mathrm{d}}$ & Hetero $^{\mathrm{e}}$ & Wt-loss ${ }^{f}$ \\
\hline KMT2D G>C p.Arg5432Gly & $0.097(193 / 1991)$ & & & \\
\hline$\# 1 \mathrm{HCC}$ & & $0.000 \pm 0.000$ & $0.00 \pm 0.00$ & \\
\hline \#2HCC & & $0.063 \pm 0.027$ & $0.13 \pm 0.05$ & \\
\hline \#3cHCC-CCA & & $0.082 \pm 0.006$ & $0.16 \pm 0.01$ & \\
\hline$\# 3 \mathrm{HCC}$ & & $0.088 \pm 0.011$ & $0.18 \pm 0.02$ & \\
\hline$\# 3 \mathrm{iCCA}$ & & $0.085 \pm 0.010$ & $0.17 \pm 0.02$ & \\
\hline TP53 C>A p.Glu204Ter & $0.096(192 / 1994)$ & & & \\
\hline$\# 1 \mathrm{HCC}$ & & $-0.009 \pm 0.009$ & $0.00 \pm 0.02$ & \\
\hline$\# 2 \mathrm{HCC}$ & & $0.262 \pm 0.043$ & $0.52 \pm 0.09$ & \\
\hline$\# 3 \mathrm{cHCC}-\mathrm{CCA}$ & & $0.191 \pm 0.015$ & $0.38 \pm 0.03$ & \\
\hline \#3HCC & & $0.310 \pm 0.017$ & $0.62 \pm 0.03$ & \\
\hline$\# 3 \mathrm{iCCA}$ & & $0.208 \pm 0.010$ & $0.42 \pm 0.02$ & \\
\hline DNMT3A C>G p.Glu426Gln & $0.094(187 / 1994)$ & & & \\
\hline$\# 1 \mathrm{HCC}$ & & $0.000 \pm 0.000$ & $0.00 \pm 0.00$ & \\
\hline \#2HCC & & $0.248 \pm 0.034$ & $0.50 \pm 0.07$ & \\
\hline$\# 3 \mathrm{cHCC}-\mathrm{CCA}$ & & $0.206 \pm 0.025$ & $0.41 \pm 0.05$ & \\
\hline \#3HCC & & $0.288 \pm 0.012$ & $0.58 \pm 0.02$ & \\
\hline$\# 3 \mathrm{iCCA}$ & & $0.236 \pm 0.032$ & $0.47 \pm 0.06$ & \\
\hline PKHD1 G>C p.leu2479Val & $0.093(185 / 1998)$ & & & \\
\hline$\# 1 \mathrm{HCC}$ & & $0.000 \pm 0.000$ & $0.00 \pm 0.00$ & 0.00 \\
\hline \#2HCC & & $0.758 \pm 0.133$ & $0.36 \pm 0.24$ & $0.64 \pm 0.24$ \\
\hline$\# 3 \mathrm{cHCC}-\mathrm{CCA}$ & & $0.335 \pm 0.039$ & $0.67 \pm 0.08$ & 0.00 \\
\hline \#3HCC & & $0.688 \pm 0.029$ & $0.46 \pm 0.06$ & $0.54 \pm 0.06$ \\
\hline$\# 3 \mathrm{iCCA}$ & & $0.460 \pm 0.013$ & $0.92 \pm 0.03$ & 0.00 \\
\hline TLR4 C>T p.His456Tyr & $0.054(79 / 1456)$ & & & \\
\hline \#1HCC & & $0.000 \pm 0.000$ & $0.00 \pm 0.00$ & \\
\hline \#2HCC & & $0.172 \pm 0.114$ & $0.34 \pm 0.23$ & \\
\hline \#3cHCC-CCA & & $0.179 \pm 0.037$ & $0.36 \pm 0.07$ & \\
\hline \#3HCC & & $0.280 \pm 0.019$ & $0.56 \pm 0.04$ & \\
\hline \#3iCCA & & $0.116 \pm 0.005$ & $0.23 \pm 0.01$ & \\
\hline
\end{tabular}

${ }^{\mathrm{a}} \mathrm{Gene}$ symbols, nucleotide changes and corresponding amino acid changes and positions are presented. ${ }^{\mathrm{b}} \mathrm{DNA}$ samples are from cancer lesions from Fig. 1. ${ }^{\mathrm{c}}$ Allele frequency (mutant coverage/total coverage) was determined by amplicon sequencing of the CCP using \#3cHCC-CCA DNA. ${ }^{\mathrm{d}}$ Allele frequency was determined by SYBR green allele-specific qPCR and is expressed as the mean \pm standard deviation. The mutant allele frequency was used for cluster analysis of cancers with the agglomerative clustering and Ward method (Fig. S4). ${ }^{\mathrm{e} T h e}$ mutant cell population was determined assuming that all mutant cells were heterozygotes and is expressed as the mean \pm standard deviation. ${ }^{\mathrm{f} I n}$ cases with an allele frequency $>0.5$ (only PKHD1 SNV), assuming that the second hit of Wt allele loss occurred in partially mutant cells (Wt-loss), populations of heterozygotes $(\mathrm{Mu} / \mathrm{Wt})$ and hemizygotes $(\mathrm{Mu} /-)$ were individually determined and presented as the mean \pm standard deviation. KMT2D, histone-lysine N-methyltransferase 2D; TP53, tumor protein p53; DNMT3A, DNA (cytosine-5')-methyltransferase 3 $\alpha$; PKHD1, polycystic kidney and hepatic disease 1; TLR4, toll like receptor 4; CCP, comprehensive cancer panel; Wt, wild-type; Mu, mutant; HCC, hepatocellular carcinoma; cHCC-CCA, combined hepatocellular-cholangiocarcinoma; iCCA, intrahepatic cholangiocarcinoma; SNV, single-nucleotide variant.

histological grade was well-differentiated [Edmondson and Steiner grade I (15)] for \#1HCC and moderately differentiated (Edmondson and Steiner grade II) for \#2HCC. \#3cHCC-CCA mainly consisted of a mixed $\mathrm{HCC} / \mathrm{iCCA}$ area and partial $\mathrm{HCC}$ and iCCA components were separately located in the same tumor nodule (Fig. 1). Immunohistochemistry for CK7 and CA19-9, known as iCCA markers (16), clearly indicated \#3HCC and \#3iCCA to be regionally separated (Figs. 1 and S1).
To perform a precise molecular analysis of cancer evolution, multiple cancerous and noncancerous regions were obtained by histological examination of FFPE tissues used in the pathological diagnostic division of the hospital, followed by dissection or LCM techniques. DNA was extracted from the dissected tissue specimens of three metachronous liver cancers, including noncancerous tissues: \#1N, \#1HCC, \#2N, $\# 2 \mathrm{HCC}$, \#3N and \#3cHCC-CCA (whole cancerous region of 
A

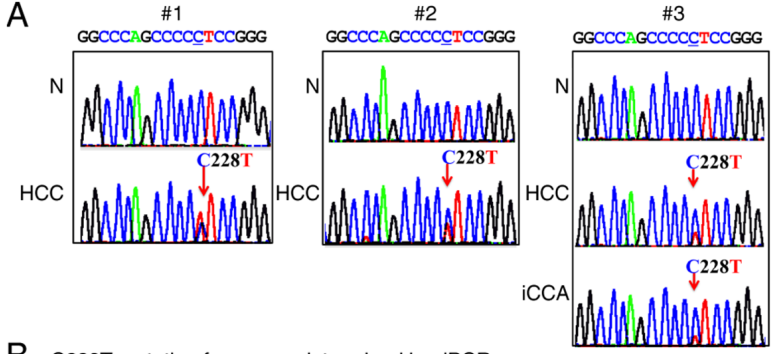

B C228T mutation frequency determined by $\mathrm{dPCR}$

\begin{tabular}{|c|c|c|c|c|c|c|c|c|c|}
\hline & $\# 1 \mathrm{~N}$ & $\# 1 \mathrm{HCC}$ & \#2N & \#2HCC & $\# 3 \mathrm{~N}$ & $\# 3 \mathrm{HCC}$ & \#3iCCA & Liver & Blood \\
\hline 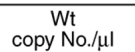 & 205.9 & 105.1 & 175.3 & 156.4 & 818.4 & 54.1 & 91.4 & 1635 & 1316 \\
\hline $\begin{array}{c}\mathrm{Mu} \\
\text { copy No./ul }\end{array}$ & 1.73 & 65.5 & 1.03 & 136.9 & 0.91 & 22.1 & 25.3 & 0 & 0 \\
\hline $\begin{array}{c}\mathrm{Mu} \\
\text { frequency (\%) }\end{array}$ & 0.83 & 38.4 & 0.58 & 46.7 & 0.11 & 29.0 & 21.7 & 0 & 0 \\
\hline
\end{tabular}

Figure 2. TERT promoter mutation C228T. (A) Sanger sequencing of the TERT promoter region. Nested PCR was performed using seven DNA samples (\#1N, \#1HCC, \#2N, \#2HCC, \#3N, \#3HCC and \#3iCCA), which were then subjected to Sanger sequencing. An arrow indicates the C228T single nucleotide variant. (B) dPCR of the C228T mutation. The numbers of $\mathrm{Mu} / \mathrm{WT} /$ both allele amplifications in each nontumorous DNA sample (\#1N, \#2N and \#3N) were as follows: 19/2429/3, 14/2233/0 and 10/8007/2, respectively. The levels in two negative control DNA samples, liver (nontumorous liver of a patient with liver metastasis of gallbladder cancer) and blood (healthy donor blood) were 0/9942/0 and 0/9318/0, respectively. TERT, telomerase reverse transcriptase; Wt, wild-type; $\mathrm{Mu}$, mutant/mutation; $\mathrm{N}$, nontumorous liver section; HCC, hepatocellular carcinoma; iCCA, intrahepatic cholangiocarcinoma; dPCR, digital PCR.

\#3). \#3HCC and \#3iCCA were extracted from LCM tissues (Fig. 1). All DNA samples were negative for HBV DNA (Fig. S2). Therefore, the three metachronous liver cancers were $\mathrm{C}$-type (HCV-positive) liver cancers.

Genetic evolution of liver cancers. DNA quality from the FFPE samples was determined by GAPDH qPCR to be 0.006 to 0.118 compared with frozen tissue DNA (set as 1). The DNA quality of \#1HCC and \#2HCC was 0.006 and 0.008 , respectively, and was $<1 / 10$ of that of \#3cHCC-CCA and \#3N (0.118 and 0.059 , respectively), which was due to the higher concentration of formalin used for tissue fixation (13). Therefore, \#3cHCC-CCA and \#3N were subjected to amplicon sequencing to determine tumor-specific nonsynonymous SNVs of all exons of 409 cancer-associated genes (Table SIII). A total of 173 SNVs were detected by Tumor-Normal Pair Analysis version 5.2 of Ion Reporter. A total of 5 nonsynonymous SNVs [histone-lysine N-methyltransferase 2D (KMT2D), tumor protein p53 (TP53), DNA (cytosine-5')-methyltransferase $3 \alpha$ (DNMT3A), polycystic kidney and hepatic disease 1 (PKHD1) and toll like receptor 4 (TLR4)] were detected in \#3HCC-CCA after filtering by the following three factors: i) Allele coverage in the nontumorous sample of 0 ; ii) total coverage in the tumor sample of $>100$; and iii) allele frequency in the tumor sample of $>0.05$ (Table SIV). The allele-specific qPCR results for these SNVs validated the presence of somatic mutations in not only \#3cHCC-CCA but also in both \#3HCC and \#3iCCA (Table I). Furthermore, all five SNVs were detected in \#2HCC but not in \#1HCC. The allele frequency of the five SNVs varied from 0.063-0.088 for KMT2D to 0.335-0.758 for PKHD1, but the frequency pattern of the five SNVs was similar among the three dissected cancer samples, \#2HCC, \#3HCC and \#3iCCA. The mutant cell populations of variant

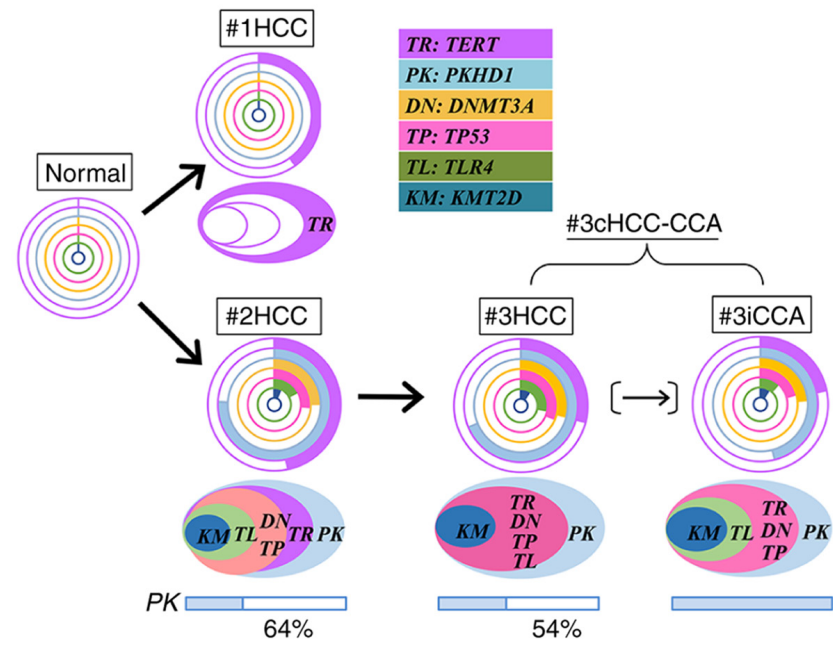

Figure 3. Schematic of the molecular evolution of three metachronous liver cancers. Different allele frequencies of six SNVs, including the TERT promoter mutation, are presented in doughnut charts and used for the cluster analysis (Fig. S4). Mutant cancer cell populations were predicted as indicated in Table I and cancer cell evolution by accumulation of somatic mutations is presented from right to left in ellipses. Bar charts indicate the population of two types of PKDH1-mutant cells: Heterozygous with SNV (colored) and hemizygous with wild-type loss as the second hit (blank); the \% population of the mutant hemizygote is also presented. There are two possibilities for \#3iCCA generation: \#3iCCA was directly generated from \#2HCC or indirectly generated from \#2HCC through $\# 3 \mathrm{HCC}$, as indicated by a narrow arrow in parentheses. SNV, single-nucleotide variant; HCC, hepatocellular carcinoma; cHCC-CCA, combined hepatocellular-cholangiocarcinoma; iCCA, intrahepatic cholangiocarcinoma; TERT, telomerase reverse transcriptase; KMT2D, histone-lysine N-methyltransferase 2D; TP53, tumor protein p53; DNMT3A, DNA (cytosine-5')-methyltransferase 3 $\alpha$; PKHD1, polycystic kidney and hepatic disease 1; TLR4, toll like receptor 4.

heterozygotes and hemizygotes were also calculated when the allele frequency was $>0.5$; the PKHD1 variant frequencies of $\# 2 \mathrm{HCC}$ and \#3HCC were 0.758 and 0.688 , respectively. For HCC cells containing the second hit of wild-type allele loss of the PKHD1 gene, variant frequencies were predicted to be 0.64 and 0.54 in the population, respectively. \#3iCCA contained no or a very low number of hemizygous cancer cells.

The TERT promoter mutation C228T but not C250T was detected in all of these cancers (\#1HCC, \#2HCC, \#3HCC and \#3iCCA). By contrast, no mutations were detected in nontumorous samples (\#1N, \#2N and \#3N) by Sanger sequencing (Fig. 2A). dPCR of the C228T mutation indicated a variable mutation frequency from 0.217 to 0.467 in the individual cancers: \#3iCCA $<\# 3 \mathrm{HCC}<\# 1 \mathrm{HCC}<\# 2 \mathrm{HCC}$ (Fig. 2B). Compared with normal liver and blood, nontumorous regions (\#1N, \#2N and \#3N) all contained TERT promoter mutations in a small number of cells (Figs. 2B and S3).

To summarize, the molecular evolution of three metachronous liver cancers was illustrated, as presented in Figs. 3 and S4, based on the phylogenetic principle that the truncal mutations present in the common ancestor are present in all descendants at higher frequencies. The different allele frequencies of six SNVs, including the TERT promoter mutation, are presented in doughnut charts in Fig. 3 and were subjected to cluster analysis (Fig. S4). Mutant cancer cell populations were predicted and presented in Table I and cancer cell evolution by accumulation of somatic mutations is illustrated from right to left in ellipses in Fig. 3. The phylogenetic relationships suggest 
the following evolutionary cancer history: i) \#1HCC was not related to \#2HCC and \#3cHCC-CCA; ii) \#2HCC was generated independently of \#1HCC in MC mode; iii) \#3cHCC-CCA originated from \#2HCC; \#2HCC already intrahepatically metastasized prior to curative resection and developed into \#3cHCC-CCA in IM mode; iv) \#3iCCA was generated directly from \#2HCC or indirectly from \#3HCC.

\section{Discussion}

In the present study, three major findings were obtained from the phylogenetic analysis of three metachronous liver cancers with three-year intervals: i) There were two modes of cancer occurrence, $\mathrm{MC}$ and $\mathrm{IM}$, in a single case; ii) it is possible that iCCA is generated from HCC in $\mathrm{CHCC}-\mathrm{CCA}$; iii) the TERT promoter mutation C228T was not only a common event in the three cancers but also an early event in the noncancerous chronic hepatitis liver without liver cirrhosis. The mutant cell population was $0.22-1.66 \%$ in chronic hepatitis liver cells, which probably existed prior to carcinogenesis. Of note, these results were obtained through the combination of two methods: i) Precise dissection of target tissue samples based on histopathology; and ii) the mutation frequency of multiple gene mutations determined by previously established allele-specific qPCR (13) and APCR protocols.

There have been several reports regarding how to discriminate between the two different mechanisms of recurrence, IM and MC $(3,4)$; early recurrence is indicative of IM and late recurrence is indicative of $\mathrm{MC}$ occurrence. The recurrence-free time is the most differentiating factor between IM and MC, and 18 months is the best cutoff time point (17). Recently, somatic SNV information has provided accurate diagnosis of MC and IM using whole-genome sequencing of multiple liver cancers, including synchronous and metachronous cancers (5). In particular, this comprehensive molecular diagnosis is powerful compared to previous techniques due to its high sensitivity and it is useful for cases with inconsistent clinicopathological diagnoses. It was also demonstrated here that mutational profiling is the most definitive approach for discriminating between IM and MC recurrence. Despite an approximate three-year interval from \#1HCC (well-differentiated) to \#2HCC (moderately differentiated) (28-month interval) and from \#2HCC to \#3cHCC-CCA (41-month interval), the former recurrence was MC and the latter was IM. SNVs specifically detected in only \#1HCC are convincing. Unfortunately, \#1HCC and \#2HCC were not subjected to NGS using a comprehensive cancer panel, but the following information supports a rational explanation for the conclusion in the absence of \#1HCC-specific SNVs. Intratumor heterogeneity and branched evolution have been reported and the evolutionary history of cancer metastasis has been established by several articles, including those by Gerlinger et al (18) and Gundem et al (19). Cancer evolutionary genomics using clinical samples from metastatic cancers, including the cancer cell fraction $(\mathrm{CCF})$ plot, indicates that ubiquitous mutations are present in all primary and metastatic (metachronous and synchronous) cancers, constructing a trunk phylogenetic tree. The CCF (the fraction of cancer cells within a sample containing a mutation) plot also indicates that the mutation frequency of truncal mutations is higher than that of branch and leaf mutations. Therefore, if \#1HCC had been the origin of \#2HCC and \#3HCC-CCA, the top $5 \mathrm{SNVs}$ detected in \#2HCC and \#3cHCC-CCA would have also been detected in \#1HCC. The top $5 \mathrm{SNVs}$ were at least ubiquitous mutations in \#2HCC and \#3HCC-CCA. Thus, truncal mutations were not detected in \#1HCC; i.e., \#1HCC has no relationship with \#2HCC. Furuta et al (5) used whole-genome sequencing analyses to demonstrate similar results for three metachronous HCCs with intervals of 21 and 30 months; the second $\mathrm{HCC}$ was MC and the third was IM from the second HCC. Yamamoto et al (20) also performed whole-exome sequencing of 41 multiple HCCs: 18 genomic IMs and 23 genomic MCs. Among 10 clinical MCs with recurrence $>2$ years after initial resection, 3 were genomic IMs. Thus, the recurrence-free time is ambiguous when attempting to discriminate MC and IM. The present study indicated that patients with MC occurrence have a lower risk of death following surgery than those with IM $(4,20)$. In the present case, the patient died at 6 months after the third operation due to multiple bone metastases and recurrent liver cancer in S4. Thus, the precise discrimination of IM and MC by cancer genomics is valuable for prognosis.

The carcinogenesis of cHCC-CCA is also debated with regard to cancer clonality. Wang et al (11), Joseph et al (21) and Xue et al (22) investigated the sequence-based molecular pathogenesis of cHCC-CCA by separately sequencing two components, HCC and iCCA, of 6, 9 and 41 cases, respectively. All cases carried ubiquitous mutations shared by HCC and iCCA, as well as substantial individual mutations, suggesting the monoclonal origin of cHCC-CCA and intratumor heterogeneity. In particular, Xue et al (22) performed not only genomic and but also transcriptomic profiling of multiple cHCC-CCA cases classified into three subtypes: Separate, combined (corresponding to the present case) and mixed. All cases were of monoclonal origin, except for 2 of 6 separate subtype cases. Both HCC and iCCA components of the combined subtype also exhibited a similar global gene expression pattern, and of note, high Nestin expression in both components was indicated to be a biomarker for $\mathrm{CHCC}-\mathrm{CCA}$. Thus, the combined type cHCC-CCA is certainly monoclonal, as in the present case. Next, the genesis of monoclonal but heterologous cHCC-CCA may be debated with respect to two mechanisms: Transdifferentiation of HCC or HPC origin $(6,8)$. Regarding the two mechanisms of monoclonal origin, Wang et al (11) suggested the HPC origin theory, whereby two components of cHCC-CCA originate from a common cell with stem cell-like features. On the other hand, Joseph et al (21) demonstrated that the genetics of cHCC-CCA are similar to those of HCC and distinct from iCCA and described a possible mechanism of transdifferentiation of partial HCC to iCCA via interplay between genetic factors and the surrounding tumor microenvironment $(23,24)$. The novel finding reported in the present study based on mutational profiles is a potential mechanism of transdifferentiation from HCC to iCCA in cHCC-CCA. The present study is the first, to the best of our knowledge, to indicate transdifferentiation from HCC to iCCA in clinical samples based on the natural history of cancer development, as it is difficult to obtain direct evidence from clinical samples. The present genetic analysis of metachronous liver cancers revealed that the iCCA component in \#3cHCC-CCA evolved from the preceding \#2HCC or \#3HCC. The former evolution, directly from \#2HCC to \#3iCCA, is more likely due to the Euclidean distance and clustering of cancers; \#3HCC-iCCA developed from IM-\#2HCC in three years, with \#2HCC 
evolving to \#3HCC and partial \#2 $\mathrm{HCC}$ transdifferentiating to \#3iCCA.

C228T is the most frequent TERT promoter mutation (25). The frequency of TERT promoter mutations in HCV-positive $\mathrm{HCC}$ is high, at $64-72 \%$, compared to that of $\mathrm{HBV}$, at $37-39 \%(26,27)$. By contrast, TERT promoter mutations are rare in iCCA $(5 \%)$ (28). In addition, considering this point of view, the iCCA component of cHCC-CCA in the present study was not similar to iCCA but was similar to HCC. The mutant cell population was predicted from the mutation frequency. The C228T mutant cells comprised $77 \%$ in \#1HCC and $93 \%$ in \#2HCC; on the other hand, they were $48 \%$ in the \#3HCC component and $43 \%$ in the \#3iCCA component. Most cancer cells in \#1HCC and \#2HCC were positive for the C228T mutation, but the mutant cell population similarly decreased in both components of \#3cHCC-CCA, resulting in similar mutant cell populations with TP53 and DNMT3A mutations. These results suggest that after cancer evolution, \#2HCC contained an early HCC clone harboring the C228T mutation but not the other mutations. The early clone was probably not metastatic and was filtered out, resulting in the absence of the early clone in \#3cHCC-CCA. Digital PCR of C228T clearly indicated no mutation in the negative control consisting of normal liver and normal blood samples but the presence of mutation signals in nontumorous tissues. This is a novel finding and suggests that small foci with TERT promoter mutations may be generated in nontumor lesions. Carcinogenesis may even be initiated in the chronic hepatitis state without cirrhosis. Recently, Kim et al (29) performed deep sequencing of various tumor-related genes, including the TERT promoter region, in regenerative nodules of liver cirrhosis, with an average sequence depth of 958; low-abundance mutations of TP53 and ARID1A were detected in independent nodules, but no TERT promoter mutation was observed in any of the 205 regenerative nodules from 10 cirrhotic livers examined. Although it is a novel finding that clonal expansion within a cirrhotic regenerative nodule occurs in the absence of TERT promoter mutation, it does not exclude the present results for the presence of TERT promoter mutation with lower frequency, 0.11 to $0.83 \%$, which was determined by dPCR based on $2,247-8,021$ amplifications. Thus, it is still possible that the TERT promoter mutation occurs followed by small subclonal expansion during chronic liver inflammation, even without cirrhosis.

Finally, the present study was a retrospective analysis of comprehensive cancer genomics by separate sequencing of each component of liver cancers, including cHCC-CCA, suggesting the usefulness not only for clonal evolution analysis but also for prognosis through the discrimination of IM or MC. In the future, targeted therapy based on cancer driver mutations may be useful.

\section{Acknowledgements}

Not applicable.

\section{Funding}

This work was supported in part by a grant from the Program for Promoting Advanced Medical Research in Nihon University School of Medicine (Tokyo, Japan; approval no. 2016).

\section{Availability of data and materials}

All the data analyzed during the present study are included in this published article. The datasets collected using the Ion AmpliSeq Comprehensive Cancer Panel are not publicly available as the patient died and it is therefore impossible to obtain consent. The patient consent for publication obtained contains the genetic analysis results but not NGS data containing the personal information, as NGS technology had not yet been included in the genetic analysis at that time consent was given. However, the dataset used for analysis in the present study is available from the corresponding author upon reasonable request.

\section{Authors' contributions}

SO, HY, YH and YN performed the experimental work. SO and MS performed pathological diagnoses of the tissue samples. YM was involved in the surgical treatment and diagnosis. SO, $\mathrm{HY}, \mathrm{HN}$ and TN performed the molecular genetic studies and analyzed the data. SO drafted the manuscript. ME designed the study and edited the manuscript. MM reviewed the study design, interpreted data and critically revised the manuscript for scientific content. ME and MM confirm the authenticity of all the raw data. All authors read and approved the final manuscript.

\section{Ethics approval and consent to participate}

The present study was approved by the Ethics Committee of Nihon University School of Medicine (Tokyo, Japan; approval no. 237-1). Written informed consent for use and analysis of samples was obtained from the patients and a healthy subject including those for control samples prior to the start of the study.

\section{Patient consent for publication}

Written informed consent for publication of data was obtained from the patient.

\section{Competing interests}

The authors declare that they have no competing interests.

\section{References}

1. Forner A, Llovet JM and Bruix J: Hepatocellular carcinoma. Lancet 379: 1245-1255, 2012.

2. Vilarinho $S$ and Calvisi DF: New advances in precision medicine for hepatocellular carcinoma recurrence prediction and treatment. Hepatology 60: 1812-1814, 2014.

3. Feo F and Pascale RM: Multifocal hepatocellular carcinoma: Intrahepatic metastasis or multicentric carcinogenesis? Ann Transl Med 3: 4, 2015.

4. Yang SL, Luo YY, Chen M, Zhou YP, Lu FR, Deng DF and Wu YR: A systematic review and meta-analysis comparing the prognosis of multicentric occurrence and vs. intrahepatic metastasis in patients with recurrent hepatocellular carcinoma after hepatectomy. HPB (Oxford) 19: 835-842, 2017.

5. Furuta M, Ueno M, Fujimoto A, Hayami S, Yasukawa S, Kojima F, Arihiro K, Kawakami Y, Wardell CP, Shiraishi Y, et al: Whole genome sequencing discriminates hepatocellular carcinoma with intrahepatic metastasis from multi-centric tumors. J Hepatol 66: 363-373, 2017. 
6. Sempoux C, Kakar S, Kondo F and Schirmacher P: Combined hepatocellular-cholangiocarcinoma and undifferentiated primary liver carcinoma. In: WHO classification of tumours of the digestive system. Bosman FT, Garneiro F, Hruban RH and Theise ND (eds). IARC, Lyon, pp260-262, 2019.

7. Nagahama Y, Sone M, Chen X, Okada Y, Yamamoto M, Xin B, Matsuo Y, Komatsu M, Suzuki A, Enomoto K and Nishikawa Y: Contributions of hepatocytes and bile ductular cells in ductular reactions and remodeling of the biliary system after chronic liver injury. Am J Pathol 184: 3001-3012, 2014.

8. Sia D, Villanueva A, Friedman SL and Llovet JM: Liver cancer cell of origin, molecular class, and effects on patient prognosis. Gastroenterology 152: 745-761, 2017.

9. Chiba T, Zheng YW, Kita K, Yokosuka O, Saisho H, Onodera M, Miyoshi H, Nakano M, Zen Y, Nakanuma Y, et al: Enhanced self-renewal capability in hepatic stem/progenitor cells drives cancer initiation. Gastroenterology 133: 937-950, 2007.

10. Fujimoto A, Furuta M, Totoki Y, Tsunoda T, Kato M, Shiraishi Y, Tanaka H, Taniguchi $\mathrm{H}$, Kawakami Y, Ueno $\mathrm{M}$, et al Whole-genome mutational landscape and characterization of noncoding and structural mutations in liver cancer. Nat Genet 48 : 500-509, 2016

11. Wang A, Wu L, Lin J, Han L, Bian J, Wu Y, Robson SC, Xue L, Ge Y, Sang X, et al: Whole-exome sequencing reveals the origin and evolution of hepato-cholangiocarcinoma. Nat Commun 9: 894, 2018.

12. Nakayama Y, Yamaguchi H, Einaga $\mathrm{N}$ and Esumi M: Pitfalls of DNA quantification using DNA-binding fluorescent dyes and suggested solutions. PLoS One 11: e0150528, 2016.

13. Einaga N, Yoshida A, Noda H, Suemitsu M, Nakayama Y, Sakurada A, Kawaji Y, Yamaguchi H, Sasaki Y, Tokino T and Esumi M: Assessment of the quality of DNA from various formalin-fixed paraffin-embedded (FFPE) tissues and the use of this DNA for next-generation sequencing (NGS) with no artifactual mutation. PLoS One 12: e0176280, 2017.

14. Ward Jr JH: Hierarchical grouping to optimize an objective function. J Am Stat Assoc 58: 236-244, 1963.

15. Edmondson HA and Steiner PE: Primary carcinoma of the liver: A study of 100 cases among 48,900 necropsies. Cancer 7 : $462-503,1954$

16. Nakanuma Y, Klimstra DS, Komuta M and Zen Y: Intrahepatic cholangiocarcinoma. In: WHO Classification of Tumours of the Digestive System. Bosman FT, Carneiro F, Hruban RH and Theise ND (eds). IARC, Lyon, pp254-259, 2019.

17. Huang Zy, Liang By, Xiong M, Zhan DQ, Wei S, Wang GP, Chen YF and Chen XP: Long-term outcomes of repeat hepatic resection in patients with recurrent hepatocellular carcinoma and analysis of recurrent types and their prognosis: A single-center experience in China. Ann Surg Oncol 19: 2515-2525, 2012.

18. Gerlinger M, Rowan AJ, Horswell S, Math M, Larkin J, Endesfelder D, Gronroos E, Martinez P, Matthews N and Stewart A: Intratumor heterogeneity and branched evolution revealed by multiregion sequencing. N Engl J Med 366: 883-892, 2012.

19. Gundem G, Van Loo P, Kremeyer B, Alexandrov LB Tubio JM, Papaemmanuil E, Brewer DS, Kallio HM, Högnäs G, Annala M, et al: The evolutionary history of lethal metastatic prostate cancer. Nature 520: 353-357, 2015.
20. Yamamoto S, Midorikawa Y, Nagae G, Tatsuno K, Ueda H, Moriyama M, Takayama T and Aburatani H: Spatial and temporal expansion of intrahepatic metastasis by molecularly-defined clonality in multiple liver cancers. Cancer Sci 111: 601-609, 2020.

21. Joseph NM, Tsokos CG, Umetsu SE, Shain AH, Kelley RK, Onodera C, Bowman S, Talevich E, Ferrell LD, Kakar S and Krings G: Genomic profiling of combined hepatocellular-cholangiocarcinoma reveals similar genetics to hepatocellular carcinoma. J Pathol 248: 164-178, 2019.

22. Xue R, Chen L, Zhang C, Fujita M, Li R, Yan SM, Ong CK, Liao X, Gao Q, Sasagawa S, et al: Genomic and transcriptomic profiling of combined hepatocellular and intrahepatic cholangiocarcinoma reveals distinct molecular subtypes. Cancer Cell 35: 932-947 e938, 2019.

23. Hill MA, Alexander WB, Guo B, Kato Y, Patra K, O'Dell MR, McCall MN, Whitney-Miller CL, Bardeesy N and Hezel AF: Kras and Tp53 mutations cause cholangiocyte- and hepatocyte-derived cholangiocarcinoma. Cancer Res 78: 4445-4451, 2018.

24. Seehawer M, Heinzmann F, D'Artista L, Harbig J, Roux PF, Hoenicke L, Dang H, Klotz S, Robinson L, Doré G, et al: Necroptosis microenvironment directs lineage commitment in liver cancer. Nature 562: 69-75, 2018.

25. Vinagre J, Almeida A, Populo H, Batista R, Lyra J, Pinto V, Coelho R, Celestino R, Prazeres H, Lima L, et al: Frequency of TERT promoter mutations in human cancers. Nat Commun 4: 2185,2013

26. Nault JC, Mallet M, Pilati C, Calderaro J, Bioulac-Sage $P$, Laurent C, Laurent A, Cherqui D, Balabaud C and Zucman-Rossi J: High frequency of telomerase reverse-transcriptase promoter somatic mutations in hepatocellular carcinoma and preneoplastic lesions. Nat Commun 4: 2218, 2013.

27. Totoki Y, Tatsuno K, Covington KR, Ueda H, Creighton CJ, Kato M, Tsuji S, Donehower LA, Slagle BL, Nakamura H, et al: Trans-ancestry mutational landscape of hepatocellular carcinoma genomes. Nat Genet 46: 1267-1273, 2014.

28. Fujimoto A, Furuta M, Shiraishi Y, Gotoh K, Kawakami Y, Arihiro K, Nakamura T, Ueno M, Ariizumi SI, Nguyen HH, et al: Whole-genome mutational landscape of liver cancers displaying biliary phenotype reveals hepatitis impact and molecular diversity. Nat Commun 6: 6120, 2015.

29. Kim SK, Takeda H, Takai A, Matsumoto T, Kakiuchi N, Yokoyama A, Yoshida K, Kaido T, Uemoto S, Minamiguchi S, et al: Comprehensive analysis of genetic aberrations linked to tumorigenesis in regenerative nodules of liver cirrhosis. J Gastroenterol 54: 628-640, 2019.

This work is licensed under a Creative Commons Attribution-NonCommercial-NoDerivatives 4.0 International (CC BY-NC-ND 4.0) License. 